\title{
Negative Emotional Valence Intensity Affects Visual Attention in the Information Encoding Process
}

\author{
Yasuhiro Takeshima ${ }^{1}$ \\ ${ }^{1}$ Department of Psychology, Doshisha University, Japan \\ Correspondence: Yasuhiro Takeshima, Department of Psychology, Doshisha University, 1-3 Tatara Miyakodani, \\ Kyotanabe-shi, Kyoto 610-0394, Japan
}

Received: December 21, 2017

Accepted: January 15, 2018

Online Published: January 17, 2018

doi:10.5539/ijps.v10n1p19

URL: http://doi.org/10.5539/ijps.v10n1p19

\begin{abstract}
We process surrounding information by orienting our attention to important objects. Our attention is often drawn towards to the objects that carry emotional information. Therefore, the functions of emotional information on visual attention have been examined extensively. Theoretically emotion comprises two dimensions, valence and arousal. Previous studies have reported that stimuli with high arousal intensity capture visual attention more strongly. However, the relationship between visual processing and negative emotional valence intensity has not investigated sufficiently. Therefore, I conducted two experiments to examine this relationship. In Experiment 1, participants performed a visual search task with the following three valence levels: neutral (control) and highand low-intensity negative emotional valence stimuli (both angry faces). Results indicated that response times for high-intensity negative emotional valence stimuli were shorter than those of the low-intensity ones. In Experiment 2, participants were asked to detect a target face among successively presented faces. Facial stimuli were the same as those used in Experiment 1. Results revealed that accuracy was higher for identifying angry faces than for neutral faces. However, performance did not differ as a function of negative emotional valence intensity. Overall, the task performance differences between negative emotional valence intensities were observed in visual search, but not in attentional blink. Therefore, negative emotional valence intensity likely contributes to the efficiency of visual information encoding.
\end{abstract}

Keywords: Emotional valence, Negative emotion, Visual search, Attentional Blink

\section{Introduction}

Daily life is replete with visual information. We extract important information efficiently from the surrounding environment by orienting our attention to objects. Attention can be captured by novelty objects (e.g., Shen \& Reingold, 2001; Wang \& Cavanagh, 1994), faces (e.g., Langton, Law, Burton, \& Schweinberger, 2008), and bodies (e.g., Downing, Bray, Rofers, \& Childs, 2004). Further, as emotion links to selective attention (e.g., Fenske \& Raymond, 2005; Raymond, Feske, \& Tavassoli, 2003), emotional stimuli also capture attention. For example, when emotional pictures are embedded within a successive visual presentation stream, target detection deficits occur more often as compared to when individuals are presented with neutral pictures (Most, Chun, \& Widders, 2005; Most, Smith, Cooter, Levy, \& Zald, 2007). Some previous researches using a visual search task with emotional stimuli as targets or distractors at search display (e.g., Öhman, Flykt, \& Esteves, 2001) have also revealed the attentional capture effects. On the other hand, Haas, Amso, and Fox (2017) presented facial emotion as priming stimuli prior to visual search display, and they found that primed threat facial stimuli (i.e., angry, surprised and fearful faces) degraded the search efficiency of individuals of high levels of social anxiety. Schmidt, Belopolsky, and Theeuwes (2015) have revealed the attentional capture of emotional stimuli using different approach. They presented neutral shape stimuli (diamonds), combined with an electrical shock for fear-conditioning, as distractors. Their results showed that the fear-conditioned distractor slowed a search more as compared to the distractor without fear association. Moreover, the presence of an irrelevant emotional picture was found to distract multi-object tracking in a task with a high level of difficulty (D'Andrea-Penna, Frank, Heatherton, \& Tse, 2017). Additionally, Peltola, Forssman, Puura, Ijzendoorn, and Leppänen (2015) have shown that 7month-old infants' attention to fearful faces at predicted their attachment security. Specifically, infants with a smaller attentional bias to fearful faces exhibited insecure attachment.

Emotion is considered to comprise two dimensions, valence and arousal (Lang, 1995). The emotional arousal 
dimension has been examined in several studies by classifying the stimuli according to their arousal rating scores (e.g., Andersen, 2005; Vogt, Houwer, Koster, Van Damme, \& Crombez, 2008). Andersen (2005) has reported that the accuracy of the second target increases for high arousal word stimuli as compared to that for moderate arousal and neutral word stimuli. Using the dot-probe paradigm, Vogt et al. (2008) have shown that high arousal pictures capture spatial attention more rapidly as compared with low arousal pictures. Sussman, Heller, Miller, and Mohanty (2013) have reported that high arousal negative distractors inhibit dot color identification, while low arousal ones facilitate it. Moreover, Lundqvist, Juth and Öhman (2014) have revealed that emotional arousal is a key factor for rapid target detection in visual search when facial expression stimuli are used (this task is called the face-in-the crowd task; also see Feldmann-Wüstefeld, Schmidt-Daffy, \& Schubö, 2011; Hansen \& Hansen, 1988; Schmidt-Daffy, 2011). Lundqvist et al. (2014) have reanalyzed the response times (RTs) of angry and happy faces as well as the arousal data from their own laboratory's studies and found a significant correlation between RTs and arousal scores. Subsequent research has also indicated that continuous arousal saliency contributes to visual search efficiency (Lundqvist, Buce, \& Öhman, 2015).

In contrast to emotional arousal, the emotional valence dimension is dealt with by qualitatively categorizing the stimuli into negative and positive stimuli. In visual search studies, rapid target detection has been reported for both angry (negative stimuli: e.g., Frischen, Eastwood, \& Smilek, 2008; Hansen \& Hansen, 1988; Horstmann \& Bauland, 2006) and happy (positive stimuli: e.g., Becker, Anderson, Mortense, Neufeld, \& Neel, 2011; Calvo \& Nummenmaa, 2008; Craig, Becker, \& Lipp, 2014) faces. Moreover, in the attentional blink (AB) paradigm (Raymond, Shapiro, \& Arnell, 1992), both negative and positive stimuli have been found to reduce the AB effect (i.e., increasing the accuracy of the second target) when these stimuli are presented as the second target (e.g., de Jong, Koster, van Wees, \& Martens, 2009; de Jong, \& Martens, 2007; Fox, Russo, \& Georgiou, 2005). Gupta, Hur, and Lavie (2015) demonstrated that the effects of an emotional distractor on letter search task performance differed for negative and positive valence stimuli depending on their perceptual load.

On the other hand, emotional valence has also been measured by continuous variables (e.g., Goeleven, Readt, Leyman, \& Verschuere, 2008; Lang, 1995). Therefore, valence scores seem to represent a distinct intensity of facial expression stimuli as compared to arousal scores. One of the few studies (Horstmann, Lipp, \& Becker, 2012) assessing the function of emotional valence intensity on visual attention by using visual searches revealed faster detection times for high-intensity stimuli. However, in this previous study, open-mouth facial stimuli were regarded as high-intensity stimuli, and closed-mouth facial stimuli were regarded as low-intensity stimuli. These results were confounded by including salient visual features outside of the emotional context (i.e., prominent white teeth). Additionally, other tasks related on visual attention (e.g., AB or dot-probe paradigm) were not be used to investigate the effects of emotional valence intensity. Thus, the effects of emotional valence intensity on visual attention have not yet been investigated adequately.

The present study examined the effects of emotional valence intensity on visual attention. However, it focused on angry faces to examine the effects of valence intensity rather than valence category (i.e., negative or positive). Additionally, happy faces are often depicted with an open mouth. Angry and happy faces have often been found to differ in terms of low-level visual features. Additionally, such differences in low-level visual features have also been observed among angry faces. Therefore, angry faces were categorized into high-valence and low-valence intensity stimuli based on each participant's rating in the present study. This categorization minimized the effects of low-level visual features by accounting for the differences in the participants' evaluation of faces as high-valence and low-valence intensity stimuli. Furthermore, the present study conducted two tasks, a visual search and an $\mathrm{AB}$ task. While visual search pertains to encoding processes (Rauschenberger \& Yantis, 2006), the AB task is related to updating functions within working memory (Vogel, Luck, \& Shappiro, 1998). Thus, the visual search engages lower-order visual processing resources as compared to the AB. The present study attempted to examine the processing stages related to emotional valence intensity by using these two tasks. Overall, the purpose of the present study was to investigate the effects of negative emotional valence intensity on visual attentional processing. Experiment 1 examined these effects on encoding, while Experiment 2 assessed them during the updating phase of working memory. The present study attempted to elucidate the function of the continuous valence factor on visual attention.

\section{Experiment 1}

This experiment compared visual target detection performance between high- and low-intensity negative emotional valence faces during a visual search task. The purpose of Experiment 1 was to assess the effects of emotional valence intensity on visual processing during information encoding. 


\subsection{Participants.}

Thirteen students ( 6 women, 7 men) participated in Experiment 1. None had been informed about the purpose of the experiment, and all reported normal or corrected-to-normal vision. All participants gave informed consent and signed a written consent form prior to participating. The ethics committee approved all experiments.

\subsection{Materials}

A total of 92 facial images $(46$ females $)$ were selected from the KDEF Database (www.emotionlab.se/resources/kdef; Lundqvist, Flykt, \& Öhman, 1998). The facial expressions of 46 images (23 females) were angry, and 46 ( 23 females) were neutral, with a front-on view. Image size was $148 \times 200$ pixels (about $3.7^{\circ}$ width $\times 5.0^{\circ}$ height of visual angle). Images were converted to grayscale and presented in the center of the screen against a gray $\left(24.07 \mathrm{~cd} / \mathrm{m}^{2}\right)$ background. A white fixation cross (about $0.8^{\circ} \times 0.8^{\circ} ; 78.17 \mathrm{~cd} / \mathrm{m}^{2}$ ) was also presented.

\subsection{Apparatus}

The stimuli were generated and controlled by means of a custom-made MATLAB program (The Mathworks, Inc.), the Cogent Graphics and 2000 toolbox (www.vislab.ucl.ac.uk/cogent.php), and a PC (GALLERIA QF940HE, Diginnos; OS: Windows 7, Microsoft). Visual stimuli were displayed on a CRT monitor (Diamondtron Flat RDF221H, Mitsubishi; resolution: $1280 \times 1024$ pixels; refresh rate: $75 \mathrm{~Hz}$ ). Participants viewed the monitor binocularly, at a distance of $70 \mathrm{~cm}$, with their heads stabilized on a chin rest.

\subsection{Procedure}

This experiment was also divided into two parts: the valence and arousal rating task and the visual search task.

During the valence and arousal rating task, participants were required to rate the valence and arousal of the 46 angry faces on a visual analog scale (VAS) from 0 (unpleasant/excited) to 1 (pleasant/calm) using 0.01 increments. During each trial, participants clicked a mouse button to initiate the presentation of a fixation cross $(520 \mathrm{~ms})$, followed by the face image. Responses were registered when participants clicked the mouse. Participants were able to view the face until their responses were registered. For each face, the valence rating was conducted first, and faces were presented in a random order.

During the visual search task, participants were required to search for a target face within a visual display. A trial sequence is shown in Figure 1. Each trial was initiated by pressing the "0" key on the keyboard. At first, a target face was presented for $2000 \mathrm{~ms}$. Participants were required to view and memorize the face. Then, a fixation cross was presented for $520 \mathrm{~ms}$, immediately followed by the visual search display. This visual search display consisted of eight faces, which were of the same gender. The eight faces were located around a fixation cross (see, Figure 1). The size of the search display was $628 \times 760$ pixels $\left(15.7\right.$ width $\times 19.0^{\circ}$ height of visual angle). The target faces included the following categories: high-intensity negative emotional valence faces, low-intensity negative emotional valence faces, and neutral (control) faces. Two faces were selected for both the high- and low-intensity negative emotional valence face categories on both genders (i.e., two high-intensity and two low-intensity on both gender), in accordance with scores from each participant during the valence and arousal rating tasks. The top two and lower two valence scores were selected in the range of arousal scores between 0.30 and 0.70 . Mean valence ratings were $0.15 \pm 0.08$ for female high-intensity negative emotional valence faces, $0.48 \pm 0.16$ for female low-intensity negative emotional valence faces, $0.14 \pm 0.10$ for male high-intensity negative emotional valence faces, and $0.49 \pm 0.15$ for male low-intensity negative emotional valence faces. Mean valence rating scores for high-intensity negative emotional valence faces were significantly lower than those of low-intensity negative emotional valence faces, regardless of gender (Female: $t(12)=7.74, p<.001, d$ $=4.09$; Male: $t(12)=6.66, p<.001, d=2.50$ ). These faces were rated as moderately arousing (female high-valence: $M=0.53 \pm 0.10$; female low-valence: $M=0.54 \pm 0.08$; male high-valence: $M=0.50 \pm 0.08$; male low-valence: $M=0.51 \pm 0.04)$. Mean arousal rating scores between the high- and low-intensity negative emotional valence faces were not significantly different in either gender (Female: $t(12)=0.53, p=.61, d=0.17$; Male: $t(12)=1.48, p=.16, d=0.48)$. Neutral faces were selected from the same individuals used for the highand low-valence angry faces. During half of the trials, the target face was included within the visual search display. If the target face was included, the expressions of distractor faces differed from those of the target face. For example, when the target was an angry face, the distractors were neutral faces. The visual search display remained on the screen until participants registered a response. Each participant responded as to whether the target face was present or not by pressing one of two keys on a numeric keypad, "1" and " 3 " for presence and absence, respectively. Both accuracy and response time (RT) were recorded. The experiment comprised 192 trials: 2 (Target: present or absent) $\times 3$ (Valence level: high, low, or control) $\times 2($ Gender: female or male $) \times 16$ 
repetitions.

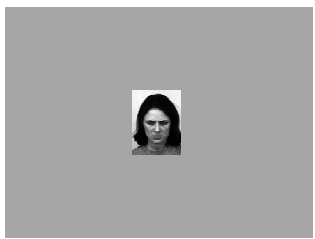

Target Display

(2000 ms)

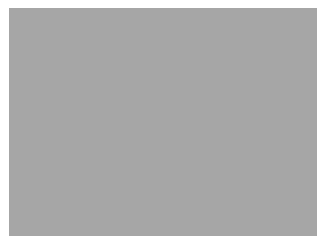

Blank

(520 ms)

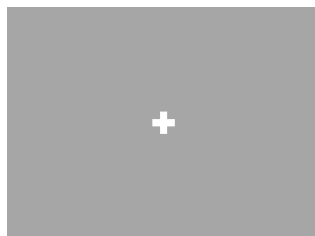

Fixation

(520 ms)

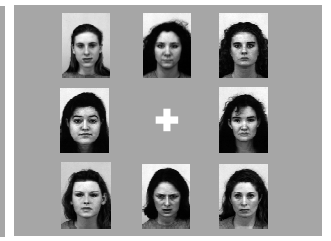

Search Display

(Until response)

time

Figure 1. Schematic representation of Experiment 1's procedure

(when an angry face was searched among neutral faces).

\subsection{Results and Discussion}

Accuracy was calculated for each condition. Additionally, average RT was computed using correct response RT data. At first, data were combined across female and male faces to investigate the general results in this experiment. Results are shown in Figure 2. Accuracy was over $90 \%$ in all conditions (high: $M=94.35 \%$, SEM = 1.43; low: $M=93.63 \%, S E M=1.33$; control: $M=90.56 \%, S E M=1.85)$. A one-way analysis of variance (ANOVA) for Valence level (3) on accuracy did not reveal a significant main effect $\left(F(2,24)=1.77, p=.19, \eta^{2}\right.$ $=.13)$. Conversely, a one-way ANOVA for Valence level (3) on RT showed a significant main effect $(F(2,24)=$ $\left.5.25, p<.05, \eta^{2}=.30\right)$. Multiple comparisons indicated that RTs were shorter for high-intensity stimuli than for the other two categories (high vs. low: $t(12)=2.27, p<.05, d=0.31$; high vs. control: $t(12)=3.56, p<.05, d=$ $0.26)$. However, a significant difference was not observed between the low-intensity and control stimuli $(t(12)=$ $0.47, p=.64, d=0.05)$.

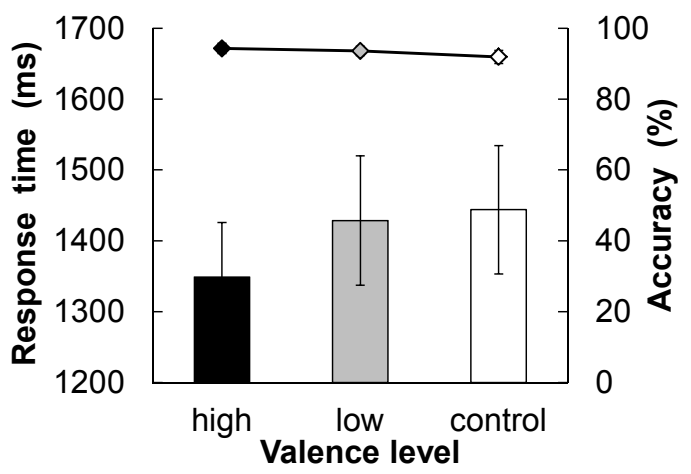

Figure 2. Overall results of Experiment 1. Bars indicate RTs, and lines indicate percent accuracy. Error bars represent standard errors of the mean $(n=13)$.

Results from Experiment 1 suggest that visual search is quicker for high-intensity emotional face targets than for low-intensity and non-emotional stimuli. The faster detection for angry faces compared with neutral faces was similar to what has been revealed in previous studies (e.g. Frichen et al., 2008; Hansen \& Hansen, 1988; Horstmann \& Bauland, 2006). Additionally, the faster detection time for high-intensity emotional face were same as the results of Horstmann et al. (2012). Because low-intensity negative emotional valence faces were detected less rapidly, these results indicate that emotional intensity may significantly impact information encoding during visual search tasks.

\section{Experiment 2}

Experiment 2 examined the effects of negative emotional valence intensity on visual target detection in an $\mathrm{AB}$ paradigm. The purpose of Experiment 2 was to assess how emotional intensity could impact the updating processes involved in visual working memory. 


\subsection{Participants}

Twenty new students (i.e. individuals who did not participate in Experiment 1: 8 women, 12 men) participated in Experiment 2. Half of the participants (4 women, 6 men) observed female facial images as targets, and the other half observed male images. All participants reported normal or corrected-to-normal vision. None of them was informed about the purpose of the experiment. All participants gave informed consent and signed a written consent form prior to their participation.

\subsection{Materials}

A total of 162 facial images ( 82 female) were selected from the KDEF Database (Lundqvist et al., 1998). A total of 46 images ( 23 female) were angry, and 68 images ( 35 female) were neutral, in a front-on view. The remaining 48 images were divided evenly into left and right profiles (24 images each; 12 females). Image face size was 295 $\times 400$ pixels (about $7.4^{\circ}$ width $\times 10.0^{\circ}$ height of visual angle) .

\subsection{Procedure}

This experiment was divided into two parts: the valence and arousal rating task and the $\mathrm{AB}$ task.

The procedure for the valence and arousal rating task was the same as in Experiment 1. However, unlike Experiment 1, all participants were required to rate only the female or male angry faces, they were presented during the $\mathrm{AB}$ task.

During the $\mathrm{AB}$ task, participants were required to discriminate a facial direction and detect a target face within a stream of face images presented in rapid succession. A trial sequence is shown in Figure 3. Each trial was initiated by pressing the "0" key on the keyboard. A fixation cross was presented for $520 \mathrm{~ms}$, immediately followed by the presentation of the rapid serial visual presentation (RSVP) stream. The RSVP stream consisted of 13 faces. Each face was presented for $120 \mathrm{~ms}$. The RSVP stream started with the presentation of a randomly chosen male or female image from the neutral face set as a distractor stimulus (without replacement). Then, the first target (T1) face was presented as either the third, fourth, or fifth face, immediately followed by a variable number of distractor faces (depending on the lag variable). The $\mathrm{T} 1$ was one of the left or right profile faces and was a different gender from the distractor faces. In a half of the trials, the second target (T2) face was presented, followed by the remainder of the distractor faces. The T2 was replaced by a distractor face for the other half of the trials. The number of lags (the number of interleaved faces between T1 and T2) was set to two, four, and six. The T2 stimuli consisted of three types according to valence level: high-intensity negative emotional valence faces, low-intensity negative emotional valence faces, and neutral faces. Each of the two faces was selected from the high and low-intensity negative emotional valence faces on both genders (i.e., two high-intensity and two low-intensity on both genders), according to scores assigned by each participant during the valence and arousal rating task. The selection criteria were nearly identical to those of Experiment 1 . Mean valence rating scores were $0.15 \pm 0.07$ for female high-intensity negative emotional valence faces, $0.50 \pm 0.06$ for female low-intensity negative emotional valence faces, $0.18 \pm 0.10$ for male high-intensity negative emotional valence faces, and $0.53 \pm 0.14$ for male low-intensity negative emotional valence faces. The mean valence rating scores for high-intensity negative emotional valence faces was significantly lower than it was for low-intensity negative emotional valence faces, regardless of gender (Female: $t(9)=13.29, p<.001, d=5.46$; Male: $t(9)=5.68, p$ $<.001, d=2.85$ ). On the other hand, these faces were rated as moderate arousal (female high-intensity: $M=0.49$ \pm 0.06 ; female low-intensity: $M=0.51 \pm 0.08$; male high-intensity: $M=0.49 \pm 0.07$; male low-intensity: $M=$ $0.48 \pm 0.10$ ). Mean arousal rating scores between the high- and low-intensity negative emotional valence faces were not significantly different for either gender (Female: $t(12)=0.56, p=.59, d=0.26$; Male: $t(9)=0.27, p$ $=.79, d=0.11)$. Neutral faces were selected from the same actors as those for high- and low-intensity negative emotional valence faces (i.e., a total of four neutral faces). The gender of the T1 and T2 faces were different from those of the distractor faces in order to make it easier to distinguish between the targets and distractors. Each participant indicated the facial direction for $\mathrm{T} 1$ stimuli and whether the $\mathrm{T} 2$ stimulus was present or not by pressing one of two keys on a numeric keypad, " 1 " and " 3 " for the left profiles/T2 face presence and right profiles/T2 face absence, respectively. For the T2 response display, the target face was presented in order to make it easier to respond. The experiment comprised 288 trials: 2 (T1: left or right $) \times 2(\mathrm{~T} 2$ : present or absent $) \times$ 3 (Valence level: high, low, or control) $\times 3$ (Lag: lag 2, lag 4, or lag 6) $\times 8$ repetitions. 


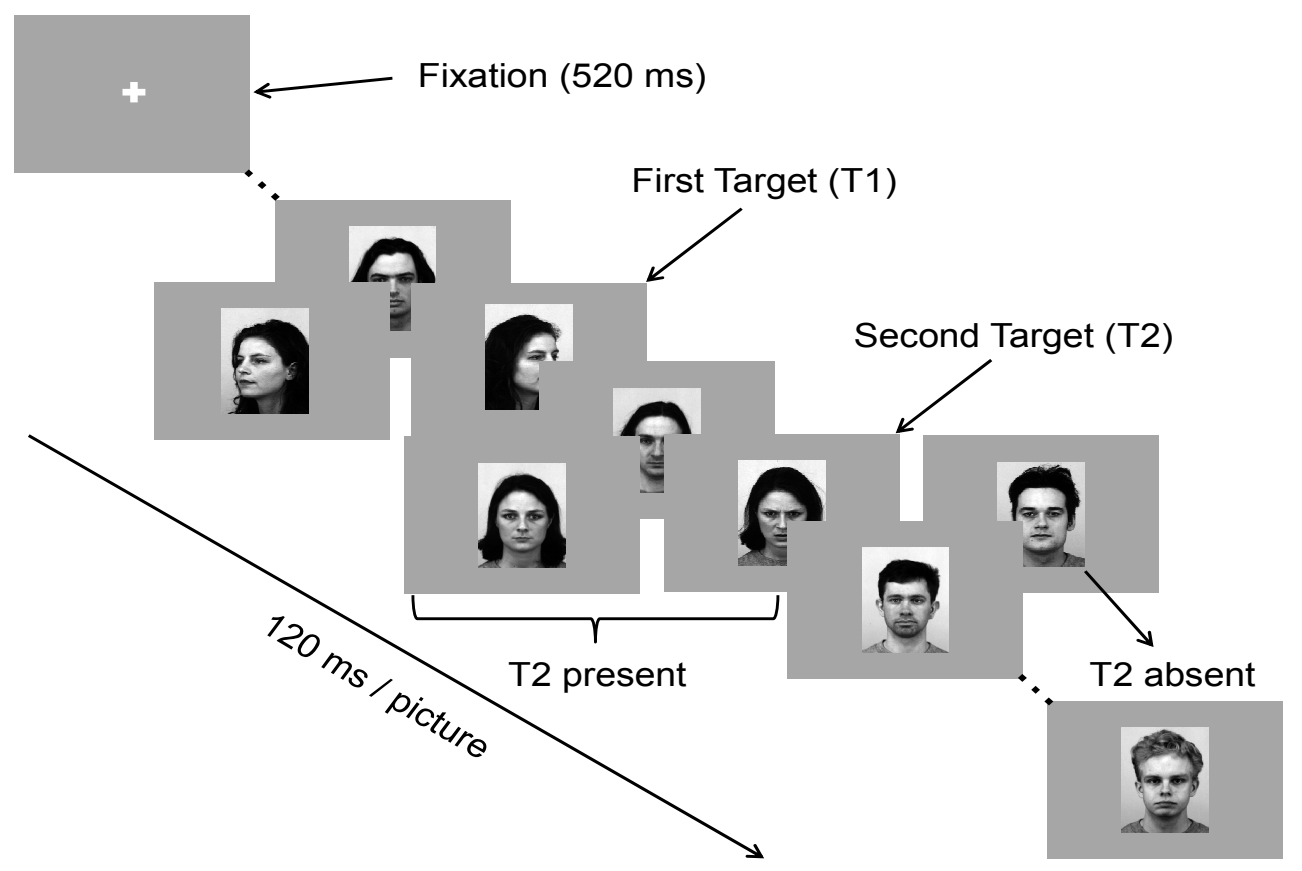

Figure 3. Schematic representation of Experiment 2's procedure. In this example, two targets were female faces, and distractors were male faces.

\subsection{Results and Discussion}

Accuracy for discriminating T1 facial direction and detecting T2 faces, with the latter contingent upon the T1 response being correct, was calculated for each condition. As in Experiment 1, data were first combined across female and male target faces to investigate the general results of this experiment. Results are shown in Figure 4. T1 discrimination was high for each condition. A two-way ANOVA with Valence level (3) $\times$ Facial direction (2) indicated no significant main effects (Valence level: $F(2,38)=0.07, p=.93, \eta_{\mathrm{p}}{ }^{2}=.00$; Facial direction: $F(1$, $\left.19)=0.23, p=.64, \eta_{\mathrm{p}}{ }^{2}=.01\right)$ and no significant interaction $\left(F(2,38)=0.29, p=.75, \eta_{\mathrm{p}}{ }^{2}=.02\right)$.

For T2 detection, a two-way ANOVA with Valence level (3) $\times$ Lag (3) was conducted. The main effects of Valence level $\left(F(2,38)=17.81, p<.001, \eta_{\mathrm{p}}{ }^{2}=.48\right)$ and $\operatorname{Lag}\left(F(2,38)=11.66, p<.001, \eta_{\mathrm{p}}{ }^{2}=.38\right)$ were significant. Multiple comparisons (Shaffer's method) for Valence level indicated that detection performance was higher for high- and low-intensity negative emotional valence faces than in control (neutral) face (high vs. control: $t(19)=4.90, p<.001, d=0.83$; low vs. control: $t(19)=4.78, p<.001, d=0.69)$. However, the accuracy was not significantly different between high- and low-valence angry faces $(t(19)=.09, p=.29, d=$ 0.18 ). Moreover, multiple comparisons for Lag indicated that detection performance was lower during lag 2 than it was for the other lag conditions (lag 2 vs. lag $4: t(19)=2.89, p<.01, d=0.57$; lag 2 vs. lag 6: $t(19)=4.44, p$ $<.01, d=0.42)$. Conversely, accuracy was not significantly different between lag 4 and lag $6(t(19)=1.71, p$ $=.10, d=0.16)$. The interaction between Valence level and Lag was not significant $\left(F(4,76)=1.07, p=.38, \eta_{\mathrm{p}}{ }^{2}\right.$ $=.05)$. 
(a)

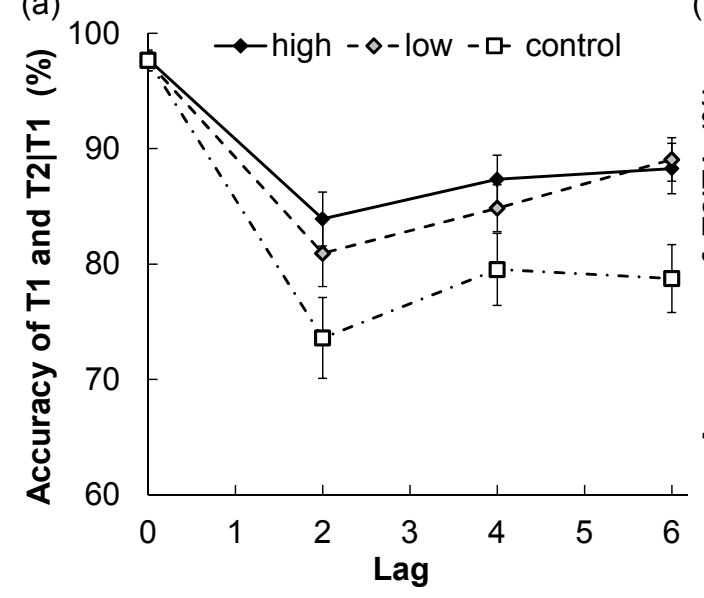

(b)

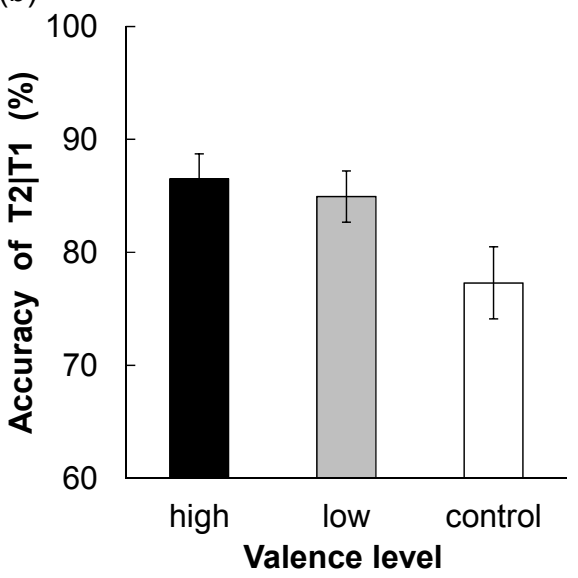

Figure 4. Results of Experiment 2. (a) T1 accuracy and T2 accuracy (given that $\mathrm{T} 1$ is correct) at each of the Valence levels and Lag conditions. Lag 0 indicates T1 accuracy. (b) T2 accuracy (given that T1 is correct) at each of the Valence level conditions. Error bars represent standard errors of the mean $(n=20)$.

Results from Experiment 2 indicated a processing advantage for angry faces in target detection compared to neutral faces within the RSVP stream. These results replicated previous studies (e.g., Bach, Schmidt-Daffy, \& Dolan, 2014; Luo, Feng, He, Wang, \& Luo, 2010). Therefore, the accurate detection of threat-related stimuli (i.e., angry and fearful faces) was observed compared to neutral stimuli (see LeDoux, 1996 for a review). Conversely, target detection performance did not differ as a function of valence intensity. Thus, emotional valence intensity did not seem to affect any updating processes within visual working memory.

\section{General Discussion}

The present study examined the relationship between negative emotional valence intensity and visual target detection. In Experiment 1, participants performed a visual search task. Visual search time was shorter for high-intensity negative emotional valence faces than for low-intensity negative emotional valence and neutral faces. However, a significant difference was not observed between low-intensity negative emotional valence faces and neutral faces. Furthermore, for Experiment 2, detection accuracy was higher for angry faces than for neutral faces, regardless of intensity. Therefore, intensity effects were observed during the visual search task but not the $\mathrm{AB}$ task.

The present study examined the effects of negative emotional valence intensity across two stages of visual information processing: encoding and working memory updating. While some previous studies suggest that visual search reflects information encoding processes (Rauschenberger \& Yantis, 2006), an AB reflects working memory updating (Vogel et al., 1998). Therefore, visual search is related to lower-order visual processing as compared with an $\mathrm{AB}$. This difference in processing stages between visual search and $\mathrm{AB}$ has been observed in event-related potential (ERP) studies. For instance, visual search evokes the N2pc component (Luck \& Hillyard, 1994), which is a negative-going deflection from 200-300 ms post-stimulus onset (Luck, 2014). Conversely, AB tasks facilitate P3 activity (Vogel et al., 1998), which is a positive-going deflection that peaks around $300 \mathrm{~ms}$ post-stimulus onset (Luck, 2014). These differential ERP latencies further confirm the lower-order nature of visual search processes relative to $\mathrm{AB}$ task performance. Furthermore, the present study revealed that negative emotional valence intensity affected target detection during visual search but not during the $\mathrm{AB}$ paradigm, suggesting that negative emotional valence intensity is more influential during lower-order information encoding processes. Negative emotional stimuli high in negative emotional valence intensity likely pose more of a threat/danger than most other stimuli. Thus, as the degree of threat increases along with increases in negative valence intensity object detection must be quick. Therefore, the present results have some ecological validity, because detection speed increased as a function of increased negative emotional valence intensity.

In the present study, visual search and $\mathrm{AB}$ paradigms were used. In the visual search task, angry and neutral faces were used, not happy faces. In many previous studies, the anger superiority effect indicates rapid target detection of angry faces among neutral and happy faces distracters (e.g., Hansen \& Hansen, 1988; Horstmann \& Bauland, 2006; Öhman, Lundqvist, \& Esteves, 2001). This study did not conduct angry target detection among happy distracters, and did not compare the target detection speed between angry and happy faces. Therefore, the 
observed search advantages of angry faces were limited. Additionally, the target face was presented before the search display presentation in the present study. Therefore, the present task might represent delayed face matching, not visual search. However, encoding the target faces is also needed in the delayed face matching task. The purpose of Experiment 1 was to compare the target detection performance between high- and low-intensity negative emotional valence stimuli in the information encoding stage. Thus, the task in Experiment 1 fulfilled the experimental purpose.

In addition, it is unclear what participants utilized to discriminate the presence or absence of $\mathrm{T} 2$ in the $\mathrm{AB}$ paradigm. In Experiment 2, T2 had various characteristics, for example, emotional category (angry or neutral), different gender from distracters, and the face identity of $\mathrm{T} 2$ itself. The results of Experiment 2 indicated that the accuracy for angry faces was higher than for neutral faces. These results might be attributed to not only emotional category, but also other characteristics. However, participants were instructed to respond whether the target was present or absent, and were presented the target face in the response display. Participants would respond to retrieve their working memory for the presented visual stimuli stream. The purpose of Experiment 2 was to compare target detection performance between high- and low-intensity negative valence stimuli in the updating process within working memory. Thus, the task in Experiment 2 also fulfilled the experimental purpose.

Previous studies using facial expression stimuli often discuss the effects of facial feature differences between facial stimuli (e.g. teeth, hair wrinkles, etc. for a review see Lundqvist et al., 2014; Savage, Becker \& Lipp, 2016). In addition, schematic faces studies have suggested that particular facial features (e.g., eyebrow or mouth) contribute to the advantageous processing for emotional facial stimuli (e.g., Fox, Lester, Russo, Bowles, Pichler, \& Dutton, 2000; Schubö, Gendolla, Meincke, \& Abele, 2006; Tipples, Atkinson, \& Young, 2002). To address this potential confound, the present study used angry faces that were missing some of these potentially salient components. Some angry faces were assigned to high-intensity emotional valence stimuli for some participants, whereas to low-intensity emotional valence stimuli for others. Therefore, the differences in facial features would be not included between high- and low-intensity negative emotional valence stimuli. On the other hand, the emotional valence intensity was not confirmed for neutral faces in the present study. Therefore, the used neutral faces might include both high- and low-intensity emotional valence stimuli. However, neutral faces were assigned randomly to distracters. Thus, the emotional valence intensity of neutral faces would affect hardly task performance.

In the present study, Caucasian faces were used with a Japanese participant sample. Thus, questions remain as to how our results apply to other racial and ethnic categories (especially when decoding in-group faces). The face perception and recognition performances were reduced for cross-race faces compared with same-race faces (e.g., Young, Hugenberg, Berstein, \& Sacco, 2012 for a review). These cross-race effects have been observed in emotional recognition (e.g., Elfenbein \& Ambady, 2003). Cross-race facial recognition requires more attention than same-race facial recognition (Hugenberg, Young, Berstein, \& Sacco, 2010). The durations of visual stimuli were shorter in Experiment 2 (each $120 \mathrm{~ms}$ ) than in Experiment 1 (until response). The perception of emotional valence might be reduced according to the interaction between cross-race face effects and the short duration of visual presentation in Experiment 2. Thus, follow-up studies should be conducted using a Japanese facial stimuli database (e.g., JAFFE database: Lyons, Akamatsu, Kamachi, \& Gyoba, 1998) to confirm the present finding.

In addition to above problem, the task difficulty might be different between angry and neutral faces in Experiment 2. In Experiment 2, participants' task was to discriminate whether the T2 faces were present or absent as Experiment 1: this task is also similar to Raymond et al. original AB paradigm). To control the task difficulty, the gender of T2 faces was always different from the gender of distractors. In addition to this control, the reference faces were presented at response display in each trial. However, participants were required to perceive all faces presented after T1 faces. The task difficulty would be high compared to the previous studies (e.g., Bach et al., 2014). According to this task difficulty, angry faces would be easy to detect compared with neutral faces regardless negative emotional valence intensity. The difference of task difficulty between angry and neutral faces might be attributed the absence of emotional valence intensity effects.

The present study examined the effects of emotional valence intensity on visual target detection in attentional processing. The two experiments suggested a relationship between emotional valence intensity and visual attentional processing. However, the present experiments have some problems. Other than those mentioned above, the number of participants was low in Experiment 1. Moreover, the present study did not measure the participants' personality traits (e.g., social anxiety or social phobia). The relationship between attentional bias for emotional stimuli and social anxiety and social phobia in both visual search and $A B$ tasks has been reported (de Jong et al., 2009; Gilboa-Schechtman, Foa, \& Amir, 1999). These participants' personality traits might affect the perceived emotional valence intensity for facial expression stimuli. In addition, this study examined only 
negative emotional valence intensity by using angry faces alone. Further studies are necessary to elucidate the effects of emotional valence intensity on visual attentional processing.

\section{Acknowledgements}

This experiment was conducted at Bunkyo Gakuin University, which is the author's former affiliation. This work was supported by a Japan Society for the Promotion of Science KAKENHI Grant Number 15H06569.

\section{References}

Andersen, A. K. (2005). Affective influences on the attentional dynamics supporting awareness. Journal of Experimental Psychology: General, 134, 258-281. https://doi.org/10.1037/0096-3445.134.2.258

Bach, D., Schmidt-Daffy, M., \& Dolan, R. (2014). Facial expression influences face identity recognition during the attentional blink. Emotion, 14, 1007-1013. https://doi.org/10.1037/a0037945

Becker, D. V., Anderson, U. S., Mortensen, D. R., Neufeld, S. L., \& Neel, R. (2011). The face in the crowd effect unconfounded: Happy faces, not angry faces, are more efficiently detected in single- and multiple-target visual search tasks. Journal of Experimental Psychology: General, 140, 637-659. https://doi.org/10.1037/a0024060

Calvo, M. G., \& Nummenmaa, L. (2008). Detection of emotional faces: Salient physical features guide effective visual search. Journal of Experimental Psychology: General, 137, 471-494. https://doi.org/10.1037/a0012771

Craig, B. M., Becker, S. I., \& Lipp, O. V. (2014). Different faces in the crowd: A happiness superiority effect for schematic faces in heterogeneous backgrounds. Emotion, 14, 794-803. https://doi.org/10.1037/a0036043

D’Andrea-Penna, G., Frank, S., Heatherton, T., \& Tse, P. (2017). Distracting tracking: Interactions between negative emotion and attentional load in multiple-object tracking. Emotion, 17, 900-904. https://doi.org/10.1037/emo0000329

De Jong, P. J., Koster, E. H., Van Wees, R., \& Martens, S. (2009). Emotional facial expressions and the attentional blink: Attenuated blink for angry and happy faces irrespective of social anxiety. Cognition \& Emotion, 23, 1640-1652. https://doi.org/10.1080/02699930802490227

De Jong, P. J., \& Martens, S. (2007). Detection of emotional expressions in rapidly changing facial displays in high- and low-socially anxious women. Behaviour Research \& Therapy, 45, 1285-1294. https://doi.org/10.1016/j.brat.2006.10.003

Downing, P., Bray, D., Rogers, J., \& Childs, C. (2004). Bodies capture attention when nothing is expected. Cognition, 93, B27-B38. https://doi.org/10.1016/j.cognition.2003.10.010

Elfenbein, H. A., \& Ambady, N. (2003). When familiarity breeds accuracy: Cultural exposure and facial emotion recognition. Journal of Personality and Social Psychology, 85, $276 \quad-290$. https://doi.org/10.1037/0022-3514.85.2.276

Feldmann-Wüstefeld, T., Schmidt-Duffy, M., \& Schubö, A. (2011). Neural evidence for the threat detection advance: Differential attention allocation to angry and happy faces. Psychophysiology, 48, 697-707. https://doi.org/10.1111/j.1469-8986.2010.01130.x

Fenske, M. J., \& Raymond, J. E. (2006). Affective influences of selective attention. Current Directions in Psychological Science, 15, 312-316. https://doi.org/10.1111/j.1467-8721.2006.00459.x

Fox, E., Lester, V., Russo, R., Bowles, R. J., Pichler, A., \& Dutton, K. (2000). Facial expressions of emotion: Are angry faces detected more efficiently? Cognition \& Emotion, 14, 61-92. https://doi.org/10.1080/026999300378996

Fox, E., Russo, R., \& Georgiou, G. (2005). Anxiety modulates the degree of attentive resources required to process emotional faces. Cognitive, Affective, \& Behavioral Neuroscience, 5, 396-404. https://doi.org/10.3758/CABN.5.4.396

Frischen, A., Eastwood, J., \& Smilek, D. (2008). Visual search for faces with emotional expressions. Psychological Bulletin, 134, 662-676. https://doi.org/10.1037/0033-2909.134.5.662

Gilboa-Schechtman, E., Foa, E. B., \& Amir, N. (1999). Attentional biases for facial expressions in social phobia: The face-in-the-crowd paradigm. Cognition and Emotion, 13, 305-318. https://doi.org/10.1080/026999399379294 
Goeleve, E., Raedt, R. D., Leyman, L., \& Verschuere, B. (2008). The karolinska directed emotional faces: A validation study. Cognition \& Emotion, 22, 1094-1118. https://doi.org/10.1080/02699930701626582

Gupta, R., Hur, Y.-J., \& Lavie, N. (2016). Distracted by pleasure: Effects of positive versus negative valence on emotional capture under load. Emotion, 16, 328-337. https://doi.org/10.1037/emo0000112

Haas, S., Amso, D., \& Fox, N. (2017). The effects of emotion priming on visual search in socially anxious adults. Cognition and Emotion, 31, 1041-1054. https://doi.org/10.1080/02699931.2016.1180281

Hansen, C., \& Hansen, R. (1988). Finding the face in the crowd: An anger superiority effect. Journal of Personality and Social Psychology, 54, 917-924. https://doi.org/10.1037/0022-3514.54.6.917

Horstmann, G., \& Bauland, A. (2006). Search asymmetries with real faces: Testing the anger-superiority effect. Emotion, 6, 193-207. https://doi.org/10.1037/1528-3542.6.2.193

Horstmann, G., Lipp, O. V., \& Becker, S. I. (2012). Of toothy grins and angry snarls-Open mouth displays contribute to efficiency gains in search for emotional faces. Journal of Vision, 12(7), 1-15. https://doi.org/10.1167/12.5.7

Hugenberg, K., Young, S. G., Bernstein, M. J., \& Sacco, D. F. (2010). The categorization-individuation model: An integrative account of the other-race recognition deficit. Psychological Review, 117, 1168-1187. https://doi.org/10.1037/a0020463

Lang, P. J. (1995). The emotion probe: Studies of motivation and attention. American Psychologist, 50, 372-385. https://doi.org/10.1037/0003-066X.50.5.372

Langton, S., Law, A., Burton, A., \& Schweinberger, S. (2008). Attention capture by faces. Cognition, 107, 330-342. https://doi.org/10.1016/j.cognition.2007.07.012

LeDoux, J. (1996). The emotional brain: The mysterious underpinnings of emotional life. NewYork, NY: Simon $\&$ Schuster.

Luck, S. J. (2014). An introduction to the event-related potential technique. (2nd ed.). London: The MIT Press.

Luck, S. J., \& Hillyard, S. A. (1994). Electrophysiological correlates of feature analysis during visual search. Psychophysiology, 31, 291-308. https://doi.org/10.1111/j.1469-8986.1994.tb02218.x

Lundqvist, D., Bruce, N., \& Öhman, A. (2015). Finding an emotional face in a crowd: Emotional and perceptual stimulus factors influence visual search efficiency. Cognition and Emotion, 29, 621-633. https://doi.org/10.1080/02699931.2014.927352

Lundqvist, D., Flykt, A., \& Öhman, A. (1998). The karolinska directed emotional faces KDEF. CD ROM from Department of Clinical Neuroscience, Psychology section, Karolinska Institutet.

Lundqvist, D., Juth, P., \& Öhman, A. (2014). Using facial emotional stimuli in visual search experiments: The arousal factor explains contradictory results. Cognition and Emotion, 28, 1012-1029. https://doi.org/10.1080/02699931.2013.867479

Luo, W., Feng, W., He, W., Wang, N-Y., \& Luo, Y-J. (2010). Three stages of facial expression processing: ERP study with rapid serial visual presentation. NeuroImage, 49, 1857-1867. https://doi.org/10.1016/j.neuroimage.2009.09.018

Lyons, M., Akamatsu, S., Kamachi, M., \& Gyoba, J. (1998). Coding facial expressions with Gabor wavelets. Proceedings, Third IEEE International Conference on Automatic Face and Gesture Recognition, 200-205. https://doi.org/10.1109/AFGR.1998.670949

Most, S. B., Chun, M. M., \& Widders, D. M. (2005). Attentional rubbernecking: Cognitive control and personality in emotion-induced blindness. Psychonomic Bulletin \& Review, 12, 654-661. https://doi.org/10.3758/BF03196754

Most, S. B., Smith, S., Cooter, A., Levy, B., \& Zald, D. (2007). The naked truth: Positive, arousing distractors impair rapid target perception. Cognition \& Emotion, 21, 964-981. https://doi.org/10.1080/02699930600959340

Öhman, A., Flykt, A., \& Esteves, F. (2001). Emotion drives attention: Detecting the snake in the grass. Journal of Experimental Psychology: General, 130, 466-478. https://doi.org/10.1037/0096-3445.130.3.466

Öhman, A., Lundqvist, D., \& Esteves, F. (2001). The face in the crowd revisited: A threat advantage with schematic stimuli. Journal of Personality and Social Psychology, 80, 381-396. https://doi.org/10.1037/0022-3514.80.3.381 
Peltola, M. J., Forssman, L., Puura, K., IJzendoorn, M. H., \& Leppänen, J. M. (2015). Attention to faces expressing negative emotion at 7 months predicts attachment security at 14 months. Child Development, 86, 1321-1332. https://doi.org/10.1111/cdev.12380

Rauschenberger, R., \& Yantis, S. (2006). Perceptual encoding efficiency in visual search. Journal of Experimental Psychology: General, 135, 116-131. https://doi.org/10.1037/0096-3445.135.1.116

Raymond, J. E., Fenske, M. J., \& Tavassoli, N. T. (2003). Selective attention determines emotional responses to $\begin{array}{lllll}\text { novel visual stimuli. } & \text { Psychological } & \text { Science, } & \text { 537-542. }\end{array}$ https://doi.org/10.1046/j.0956-7976.2003.psci_1462.x

Raymond, J. E., Shapiro, K. L., \& Arnell, K. M. (1992). Temporary suppression of visual processing in an RSVP task: An attentional blink? Journal of Experimental Psychology: Human Perception and Performance, 18, 849-860. https://doi.org/10.1037/0096-1523.18.3.849

Savage, R., Becker, S., \& Lipp, O. (2016). Visual search for emotional expressions: Effect of stimulus set on anger and happiness superiority. Cognition and Emotion, 30, 713-730. https://doi.org/10.1080/02699931.2015.1027663

Schmidt, L., Belopolsky, A., \& Theeuwes, J. (2015). Attentional capture by signals of threat. Cognition and Emotion, 29, 687-694. https://doi.org/10.1080/02699931.2014.924484

Schmidt-Daffy, M. (2011). Modeling automatic threat detection: Development of a face-in-the-crowd task. Emotion, 11, 153-168. https://doi.org/10.1037/a0022018

Schubö, A., Gendolla, G. H. E., Meinecke, C., \& Abele, A. E. (2006). Detecting emotional faces and features in a visual search paradigm: Are faces special? Emotion, 6, 246-256. https://doi.org/10.1037/1528-3542.6.2.246

Shen, J., \& Reingold, E. M. (2001). Visual search asymmetry: The influence of stimulus familiarity and low-level features. Perception \& Psychophysics, 63, 464-475. https://doi.org/10.3758/BF03194413

Sussman, T. J, Heller, W., Miller, G. A., \& Mohanty, A. (2013). Emotional distractors can enhance attention. Psychological Science, 24, 2322-2328. https://doi.org/10.1177/0956797613492774

Tiplles, J., Atkinson, A. P., \& Young, A. W. (2002). The eye frown: A salient social signal. Emotion, 2, 288-296. https://doi.org/10.1037//1528-3542.2.3.288

Vogel, E. K., Luck, S. J., \& Shapiro, K. L. (1998). Electrophysiological evidence for a postperceptual locus of suppression during the attentional blink. Journal of Experimental Psychology: Human Perception and Performance, 24, 1656-1674. https://doi.org/10.1037/0096-1523.24.6.1656

Vogt, J., Houwer, J., Koster, E., Damme, S., \& Crombez, G. (2008). Allocation of spatial attention to emotional stimuli depends upon arousal and not valence. Emotion, 8, 880-885. https://doi.org/10.1037/a0013981

Wang, Q., \& Cavanagh, P. (1994). Familiarity and pop-out in visual search. Perception \& Psychophysics, 56, 495-500. https://doi.org/10.3758/BF03206946

Young, S. G., Hugenberg, K., Bernstein, M. J., \& Sacco, D. (2012). Perception and motivation in face recognition: A critical review of theories of the cross-race effect. Personality and Social Psychology Review, 16, 116-142. https://doi.org/10.1177/1088868311418987

\section{Copyrights}

Copyright for this article is retained by the author(s), with first publication rights granted to the journal.

This is an open-access article distributed under the terms and conditions of the Creative Commons Attribution license (http://creativecommons.org/licenses/by/4.0/). 